\title{
Double emulsions (W/O/W emulsions): Encapsulation of Plant Biactives
}

\author{
Jelena Mudrić ${ }^{1, *}$, Katarina Šavikin ${ }^{1}$, Svetlana Ibrić ${ }^{2}$, And Jelena Đuriš² \\ ${ }^{1}$ Institute for Medicinal Plants Research "Dr. Josif Pančić", Tadeuša Košćuška 1, 11000 Belgrade, Serbia \\ ${ }^{2}$ University of Belgrade, Faculty of Pharmacy, Department of Pharmaceutical Technology and Cosmetology, Vojvode Stepe 450, 11221 Belgrade, Serbia \\ *Corresponding author:jmudric@mocbilja.rs
}

Received: November 5, 2019

Accepted: November 20, 2019

Published on-line: November 25, 2019

Published: December 25, 2019

\begin{abstract}
This article describes the preparation, characterization, and application of W/O/W emulsions, with emphasis on the encapsulation of plant bioactives. The main limitations preventing commercialization of double emulsions with plant bioactive substances, used for the preparation of food, nutraceuticals and pharmaceuticals for oral administration, are low thermodynamic stability and the limited range of the available lipophilic emulsifiers. In that regard, strategies for stability improvement of $\mathrm{W} / \mathrm{O} / \mathrm{W}$ emulsions with bioactive substances are highlighted.
\end{abstract}

Key words: double emulsions, plant bioactives encapsulation, multiple emulsions, target delivery, sustained delivery systems

http://dx.doi.org/10.5937/leksir1939076M

\section{INTRODUCTION}

Emulsions are heterogeneous dispersion of two immiscible liquids, with one of the liquids being dispersed as small spherical droplets in the other. Emulsions can be classified as two-phase dispersions, simple emulsions, such as oil-in-water $(\mathrm{O} / \mathrm{W})$ or water-in-oil $(\mathrm{W} / \mathrm{O})$ or complex multi-phase dispersions known as multiple emulsions. Multiple emulsions can be divided into four types: single-cored, multi-cored, Janus and multiple compartments. Single-cored emulsion depending on the number of phases (two, three, four and five phases) can be divided into double, triple, quadruple, and quintuple drops (Figure 1) (Vladisavljević et al., 2017). Double emulsion or "emulsions of emulsion "is emulsion in which primary emulsion is further dispersed in another liquid (continuous phase) (Benichou et al., 2004).

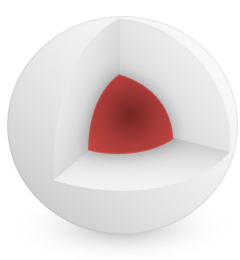

(a)

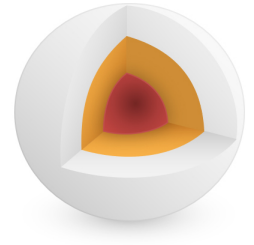

(b)

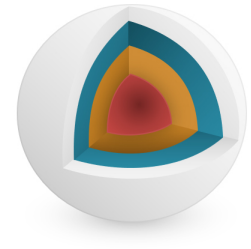

(c)
Fig. 1. Types of single-cored emulsions; a) double emulsion b) triple emulsion c) quadruple emulsion

Commonly, water-in-oil-in-water $(\mathrm{W} / \mathrm{O} / \mathrm{W})$ are used, but in some specific cases, oil-in-water-in-oil $(\mathrm{O} / \mathrm{W} / \mathrm{O})$ emulsions are prepared. Double emulsions $(\mathrm{W} / \mathrm{O} / \mathrm{W})$ are complex systems that consist of a inner $(\mathrm{W} / \mathrm{O})$ emulsion dispersed in a second continuous water phase. These emulsions $(\mathrm{W} / \mathrm{O} / \mathrm{W})$ are systems with a middle oil layer, which acts as a liquid membrane (Figure 2).
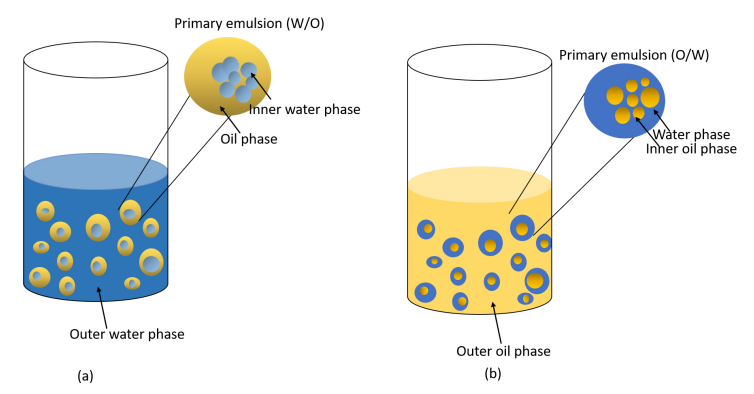

Fig. 2. Schema of a) $\mathrm{W} / \mathrm{O} / \mathrm{W}$ and $\mathrm{b}) \mathrm{O} / \mathrm{W} / \mathrm{O}$ emulsions

This approach has great potential for encapsulation of plant bioactives, due to the ability of these systems to encapsulate hydrophilic and lipophilic substances (separately and simultaneously) (Iqbal et al., 2015). However, commercial applications remain challenging due to lack of stability.

The aim of this work is to offer a review of methods for the preparation, application and strategies for stability improvement of $\mathrm{W} / \mathrm{O} / \mathrm{W}$ double emulsions with bioactive substances used for the preparation of food, nutraceuticals, and pharmaceuticals for oral administration. 


\section{METHODS FOR PREPARATION}

Traditionally double emulsions are prepared in two ways, by single-step emulsification or by two-step emulsification procedure. The one-step method involves heating of an emulsion composed of a nonionic emulsifier, or a mixture of different emulsifiers, which leads to phase inversion and the formation of multiple emulsion (Lamba et al., 2015). More often, double emulsions are formulated by a two-stage emulsification process. In the first step, the so-called inner $(\mathrm{W} / \mathrm{O})$ emulsion is formed by using a lipophilic emulsifier under intense homogenization. In the next step, the primary $\mathrm{W} / \mathrm{O}$ emulsion is dispersed in an aqueous (outer) phase using a hydrophilic emulsifier under lower shear (Akhtar et al., 2014).

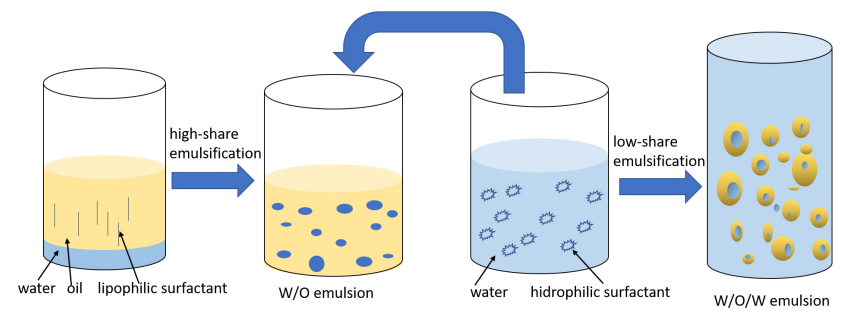

Fig. 3. $\mathrm{W} / \mathrm{O} / \mathrm{W}$ double emulsion two steps preparation process

Various methods can be employed for the formation of double emulsions, such as high-pressure homogenizers, rotorstator homogenization, ultrasound emulsification, microfluidic emulsification and membrane emulsification (van der Graaf et al., 2005; Vladisavljević et al., 2017). Stirring equipment, colloid mills, homogenizers and ultrasonics are industrially applied, but due to the high shear stress that is produced in these systems, it is not easy to control the mean size of the emulsion droplets and this results in poor control over droplet size. Moreover, high shearing stress can reduce the functionality of temperature and high shear stress-sensitive compounds (Berendsen, 2014).

In the last two decades, novel emulsification techniques (membrane emulsification, microchannel emulsification, ink-jet printing and numerous microfluidic processes) are used in order to form emulsions with uniform droplet size. In these techniques, low pressure is used to disperse the inner phase through microchannels or membrane pores in the continuous phase (Vladisavljević et al., 2012). These strategies for emulsion preparation are suitable for the production of droplets with uniform size. Advantages of preparation of an emulsion with the uniform droplet size are numerous: the content of the surfactant is lower, active ingredient/flavor is uniformly distributed, entrapment efficiency is higher and reproducibility is adequate (Lamba et al., 2015). Moreover, microfluidic junctions and flow focusing devices are able to generate emulsion droplets with controlled size, shape and internal structure (Vladisavljević et al., 2012). However, these techniques are time-consuming and the production rate is low. Therefore, they are currently used only for the production of high valuable products like drug delivery systems (van der Graaf et al., 2005; Vladisavljević et al., 2017). On the other hand, in the food industry conventional methods are generally used because of the lower price of the final product and high production scale (Vladisavljević et al., 2017).

\section{APPLICATION}

Application of multiple emulsions has been investigated in various fields such as separation technology, chemistry, encapsulation of hydrophilic/lipophilic molecules in the cosmetic, pharmaceutical or food industry (Patravale and Mandawgade,

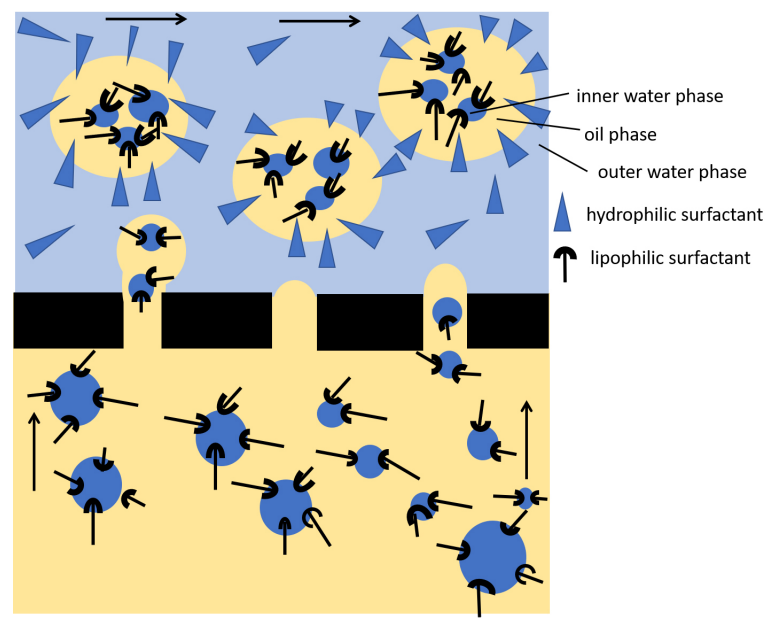

Fig. 4. Production of $(\mathrm{W} / \mathrm{O} / \mathrm{W})$ double emulsions by a membrane emulsification. The arrows are showing the fluid flow direction (redrawn from the van der Graaf et al. (2005))

2008; Schuch et al., 2013). Plant bioactives are encapsulated in double emulsions in numerous cases such as:

- The molecule encapsulated in the inner phase has to pass over several layers before it is available for absorption. The release rate is controlled by the ability of the encapsulated molecule to diffuse through different barriers. Consequently, double emulsions can be used as controlled and sustained delivery systems (Bhatia et al., 2013).

- Absorption of poorly bioavailable molecules can be improved by formulating double emulsions. It is reported that these systems are used for encapsulating bioactive compounds in order to avoid degradation in the liver and intestine. Moreover, emulsions are absorbed almost completely via lymphatic ductus (Paul et al., 2013).

- Targeting the right place at the right time is important in pharmacotherapy, especially when it is crucial to enhance the therapeutic effect and/or to reduce adverse effects. Multiple emulsions have demonstrated great potential for targeting organs (e.g. brain, liver, lungs) through the lymphatic system (Ashjari et al., 2012; Cortesi and Esposito, 2010; Dluska et al., 2017). On the other hand, stealth multiple emulsions are used in order to avoid reticuloendothelial system (RES) and RES rich organs (liver, splen) from the cytotoxic activity of the bioactive compound (Talegaonkar and Vyas, 2005).

- Microparticulate and nanoparticulate systems are extensively investigated. Double emulsions are used as intermediate products in preparation of novel systems such as polymeric microspheres and microcapsules, coreshell microparticles and gel microbeads and solid-lipid nanoparticles or microparticles (Cortesi and Esposito, 2010; Salazar-Miranda et al., 2016).

- The unpleasant taste of bioactive compounds can be minimized or eliminated by formulation of double emulsions. It is important to entrap the bioactive compound in the inner phase of double emulsion and to disable its release during the product storage or its presence in the mouth (Garti et al., 1983).

- Reducing lipid content and providing products with a healthier lipid profile. Double (W/O/W) emulsion can be used for reducing lipid content because part of the lipid material is replaced by an inner water phase. Moreover, a healthier lipid profile can be achieved by choice of most 
appropriate lipid phase which is in line with health nutrient recommendation (cholesterol-free, the optimal ratio between n-6: $n-3$ polyunsaturated fatty acids) (CarrilloNavas et al., 2012; Chung et al., 2016).

- Manufacturing products with lower content of sodium (salt), since the reduced intake of sodium, can improve health outcomes. Formulation of $\mathrm{W} / \mathrm{O} / \mathrm{W}$ emulsions with a lower amount of sodium and the desired taste is a challenging task. It has been reported that only the salt presented in the outer water phase of $\mathrm{W} / \mathrm{O} / \mathrm{W}$ emulsion will be perceived, while salt in the inner water phase will not contribute (Jiménez-Colmenero, 2013).

- Encapsulation of sensitive bioactive compounds (vitamins, minerals, amino acids, polyphenolic compounds, probiotics) in double emulsions in order to protect them from environmental stresses during production, storage, transport and utilization (Jiménez-Colmenero, 2013).

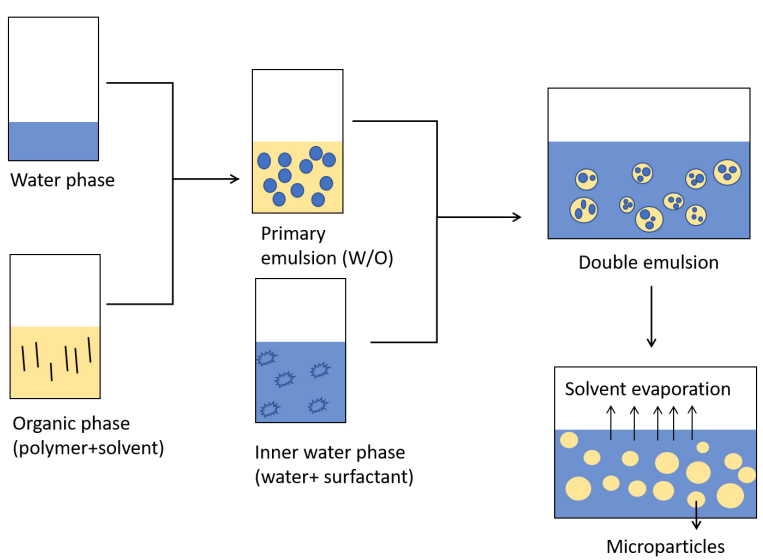

Fig. 5. Scheme of microparticles preparation by the double emulsion solvent evaporation technique (redrawn from the Giri et al. (2013))

\section{COMPOSITION AND METHODS FOR IMPROVING STABILITY}

Double emulsions contain two thermodynamically unstable interfaces, $\mathrm{W} / \mathrm{O}$ interface of primary emulsion and $\mathrm{O} / \mathrm{W}$ interface of the continuous phase. Consequently, the major limitation in the case of double emulsions is low thermodynamic stability. Mechanisms which lead to the destabilization of $\mathrm{W} / \mathrm{O} / \mathrm{W}$ emulsions are (Schmidts et al., 2010):

- internal aqueous droplets coalescence,

- the oil droplets coalescence,

- rupture of the oil film resulting in the loss of the internal aqueous droplets,

- passage of the water and water-soluble substances through the oil layer between both water phases.

In order to prepare stable $\mathrm{W} / \mathrm{O} / \mathrm{W}$ emulsions, it is important to understand the influences of various formulation and process factors and their interactions. The interfacial tension between the oil and water phase can be reduced by the addition of emulsifier(s). The choice of emulsifier(s) and the respective concentration(s) is a very important step in the formulation of stable primary $(\mathrm{W} / \mathrm{O})$ as well as multiple emulsion $(\mathrm{W} / \mathrm{O} / \mathrm{W})$. Due to the complex nature of multiple emulsions at least two emulsifiers, one hydrophilic and one lipophilic

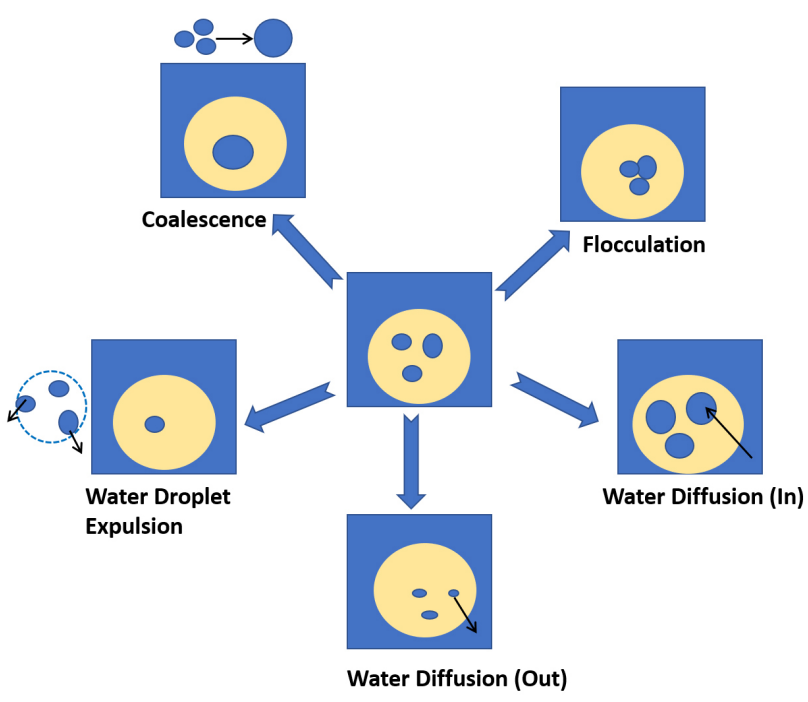

Fig. 6. Instabilities responsible for the destabilization of double (W/O/W) emulsions (redrawn from the McClements et al. (2009))

emulsifier, are required. Non-ionic polymeric emulsifiers are preferred over other types of emulsifiers, because of better stability, higher yield, and the ability to control the release of the bioactive compounds. Furthermore, it is suggested that more stable multiple emulsions are formulated when hydrophilic and lipophilic emulsifiers with the same length of the hydrocarbon chain are used (e.g. Span 80 and Tween 80). It is also reported that naturally occurring macromolecules, such as proteins and polysaccharides, are able to additionaly stabilize multiple emulsions through electrostatic and steric effects (Raviadaran et al., 2018).

Lipophilic emulsifier with hydrophilic-lipophilic balance (HLB) between 6 and 16 is necessary for stabilization of the $\mathrm{O} / \mathrm{W}$ interface. Hydrophilic emulsifiers used for preparation of double emulsions with plant bioactives are whey or soy protein isolates, sodium caseinate, bile acid, Tween 80 (polyoxyethylene sorbitan monooleate, polysorbate 80 ), Tween 20 (polyoxyethylene sorbitan monolaurate, polysorbate 20), $\beta$ lactoglobulin isolated from whey protein isolates, mixture of protein and polysaccharide (whey protein complex with metoxyl pectin and $\kappa$-carrageenan) and conjugates of protein and polysaccharide formed through a Millard-type reaction (whey protein-dextran, caseinate-dextran, ultrafiltrated whey protein complex-pectin, ovalbumin-dextran) (Lamba et al., 2015).

Lipophilic emulsifiers are emulsifiers with HLB values between 2 and 7 and are used for stabilization of the $\mathrm{W} / \mathrm{O}$ interface. Polyglycerol ester of polyricinoleic acid (PGPR) is commonly used as lipophilic emulsifier and it is reported that optimal concentration of PGPR required for stabilization of $\mathrm{W} / \mathrm{O} / \mathrm{W}$ is between 4 and $6 \%$. Concentration of the PGPR in $\mathrm{W} / \mathrm{O} / \mathrm{W}$ emulsion was reduced when the combination of PGPR $(2 \%)$ and sodium caseinate $(0.5 \%)$ was used (Lamba et al., 2015). Higher concentration of PGPR leads to the formation of droplets with a smaller average size, but when PGPR is incorporated at moderate levels in food products it is identified by consumers as an unpleasant taste (Artiga-Artigas et al., 2019; Muschiolik and Dickinson, 2017). Span 80 (sorbitan monooleate) and the mixture of glycerol monooleate and lecithin in equal amounts are also used as lipophilic emulsifiers (Lamba et al., 2015). The limited range of food-grade lipophilic emulsifiers is an issue that limits versatility of multilple emulsions applications in the case of food products, nutraceuticals and pharmaceuticals for oral administration (Muschiolik and Dickinson, 2017). 
The concentration of hydrophilic and lipophilic emulsifiers should be optimized in order to prepare stable $\mathrm{W} / \mathrm{O} / \mathrm{W}$ emulsion. Excess amount of hydrophilic emulsifier can cause the solubilization of lipophilic emulsifier. On the other hand, the excess amount of lipophilic emulsifiers can result in the formation of simple $\mathrm{O} / \mathrm{W}$ emulsion. The weight ratio of the hydrophilic to lipophilic emulsifier should be between 2 to 20 in order to obtain acceptable encapsulation of $\mathrm{W} / \mathrm{O}$ emulsion in $\mathrm{W} / \mathrm{O} / \mathrm{W}$ multiple emulsion. Furthermore, it is reported that hydrophilic emulsifiers may increase the movement of molecules from the inner water phase to the external water phase. In that regard, the concentration of hydrophilic emulsifiers should be as low as possible (Raviadaran et al., 2018).

Different lipid phases are used for the preparation of multiple emulsions for various purposes. Mineral oils (liquid paraffin and medium-chain triglycerides) and vegetable oil (soybean, sunflower, olive, sesame, corn, castor oil) can be used in the formulation of multiple emulsions (Raviadaran et al., 2018). It is reported that multiple emulsions with mineral oils are characterized with better stability and yield in comparison to the multiple emulsions with vegetable oils. However, vegetable oils are preferred in the case of nutraceutical products, since the rate of excretion of mineral oils is low (Lamba et al., 2015). The nature of the lipid phase affects the viscosity of emulsion and diffusion of the bioactive compound(s) via the lipid membrane. The use of highly viscous lipid phase is suggested in order to prevent diffusion of water and water-soluble substances between the inner and outer water phase (Schmidts et al., 2009).

The ratio between the dispersed $(\mathrm{W} / \mathrm{O})$ and continuous phase $(\mathrm{W} / \mathrm{O} / \mathrm{W})$ is known as the phase volume ratio. It is reported that a high phase volume ratio may lead to destabilization of emulsion due to coalescence. Generally, it is reported that the droplet size increases with the increase of phase volume ratio. Thus, the optimal ratio between $\mathrm{W} / \mathrm{O}$ and $\mathrm{W} / \mathrm{O} / \mathrm{W}$ is in the range from 22 to $40 \%$ (Raviadaran et al., 2018).

Other excipients, such as thickening agents and osmolarity regulators, are added in the formulation of multiple emulsions. Increasing the viscosity of the inner and external water phase prevents coalescence and movement of water between the external and internal phases. Furthermore, the gelation of the internal phase improves encapsulation efficiency. Increase in viscosity leads to a decrease in droplet size. Gelatine, alginate, xanthan gum, gum arabic and pectin are commonly used as gelling or thickening agents (Surh et al., 2007). Osmolarity regulators, such as sodium chloride and carbohydrates, are crucial for control of the osmotic gradient between the internal and external phases. The addition of electrolytes increases the effect of the emulsifier and stability of the emulsion. It is reported that the main effect of osmolarity regulator is related to the resistance of the oil film to coalescence, by decreasing the interfacial tension and increasing the adsorption density (Raviadaran et al., 2018). The high concentration of electrolytes may lead to swelling of the primary emulsion. On the other hand, a low concentration of electrolytes can cause shrinking. Consequently, the concentration of electrolytes in the internal phase of the emulsion should be optimized (Lamba et al., 2015; Mezzenga et al., 2004).

Moreover, it is necessary to optimize the critical process parameters such as sheare, time and temperature. In the case of the two-step emulsification method, high shear is used for the preparation of primary emulsion and gentle stirring is used for the preparation of the multiple emulsion. If the high shear is applied in the second step, a simple emulsion will be made instead of a multiple emulsion. Furthermore, if the low shear rate is applied during the first emulsification step, emulsion with bigger droplets and a predisposition for coalescence will be formed. A high shear used for a longer period will result in air accumulation. In order to prepare multiple emulsion with satisfactory characteristics, it is important to consider the temperature stability of bioactive compounds and a melting point of oils.

Drying of multiple (double) emulsions is reported as a predominant strategy for the enhancement of long-term stability. The most commonly used methods for transformation of liquids into powders are spray drying and freeze-drying. These methods are simple, continuous and economical. Properties of the dried emulsion (flowability, wettability, porosity, cohesion) are influenced by the composition of the parent multiple emulsion. It is reported that particles with uniform size with nearly spherical shape and appropriate flowability are obtained by using spray drying and freeze-drying (Lamba et al., 2015). Furthermore, increased thermal stability and minimized oxidation of the lipid phase components are additional advantages of described methods. In order to select the appropriate drying method for the multiple emulsion, it is crucial to consider glass transition temperature of $(\mathrm{Tg})$ encapsulated compounds, because of the stability of final product. Amorphous glassy materials are preferred because they are more stable due to the low water mobility and a consequently lower rate of oxygen diffusion and bioactive compounds stability (Champagne and Fustier, 2007; Lamba et al., 2015). In the case of freeze-drying, cryoprotectants are used in order to protect emulsion from physical and chemical degradation induced by temperature stress. In that regard, it is important to choose the adequate cryoprotectant (trehalose, sucrose, maltose, glucose, mannitol) at its optimal concentration. The ideal concentration of cryoprotectants should be determined by analyzing factors such as the formulation of the multiple emulsion, cooling rate and the freezing temperature (Morais et al., 2016).

\section{DOUBLE EMULSIONS CHARACTERIZATION}

In order to improve the efficiency of formulations with plant bioactives, it is important to investigate organoleptic, rheological, morphological as well as in vitro release and encapsulation efficiency of the formulated double $(\mathrm{W} / \mathrm{O} / \mathrm{W})$ emulsions. Organoleptic properties (color, taste and smell) are physical properties of the prepared emulsion which are usually investigated immediately upon preparation and during storage. Changes in any of these properties imply emulsion instability. Liquefaction is also an organoleptic property, which means that the water from the inner phase is transferred to the outer water phase of the double emulsion. Furthermore, liquefaction can be the cause of phase separation. Monitoring of $\mathrm{pH}$ can contribute to understanding of reactions which are leading to destabilization of multiple emulsions (Cortesi and Esposito, 2010).

Droplets or particles size is described by the mean particle size and particle size distribution. Small particle size of the primary emulsion is usually predictor of the appropriate stability. However, the inner phase droplets with the very small size lead to the increase in the surface area and consequently surface tension increases and potentially emulsion is destabilized (Lamba et al., 2015). The size of the multiple emulsion droplets as well as the size of the droplets of the inner water phase can be measured by the optical microscopy. Based on multiple emulsion droplet size, multiple emulsions can be described as coarse $(>3 \mu \mathrm{m})$, fine $(1-3 \mu \mathrm{m})$ and micro-multiple emulsion $(<1 \mu \mathrm{m})$ (Aserin, 2008). For the structure analysis different microscopies are used: transmission electron microscopy, scanning electron microscopy, scanning transmission electron microscopy, an optical and confocal microscopy (Cortesi and Esposito, 2010). Furthermore, nuclear magnetic resonance spectroscopy is technique employed in order to investigate the geometrical structure as well as the composition of double emulsions (Bernewitz et al., 2013). 
The zeta potential is described as the net electrical potential at the particle surface. Generally, emulsions with the zeta potential higher than the absolute value of $25 \mathrm{mV}$ are stable. Increase of repulsive forces and stability improvement is in correlation with the increase of zeta potential of multiple emulsion (Lamba et al., 2015). Rheological analyses are very important for shelf life and stability prediction. Processing (mixing, pumping, heating, cooling) and handling activities are influenced by the viscosity of multiple emulsions. Determination of shear stress, shear rate and apparent viscosity are performed by using dynamic shear rheometer or viscometer (Pal, 2011).

The encapsulation efficiency and in vitro release studies are parameters which are used to evaluate the quality of the multiple emulsion. The quality of the multiple emulsions depends on how efficiently the bioactive ingredient is entrapped in the inner phase of emulsion. To measure the encapsulation efficiency (EE) of the multiple emulsion, amount or percentage of encapsulated bioactive compound is calculated indirectly by estimating the amount of unencapsulated bioactive compound (UBC) present in the outer phase, according to the equation presented below (Cortesi and Esposito, 2010).

\section{$\mathrm{EE}(\%)=\frac{(\text { Total bioactive amount }- \text { UBC amount }) \times 100}{\text { Total amount of drug }}$}

Release of the bioactive compound from the multiple emulsion inner phase can be estimated by using conventional dissolution apparatus, such as rotating paddle apparatus (Vasiljevic et al., 2006) or usually by dialysis method. Appropriate media and sufficient volume should be used and sink conditions should be maintained. Samples are withdrawn at different time intervals and cumulative bioactive compound concentration can be estimated. Stability testing of double emulsions is usually performed by evaluating the influence of various factors (humidity, temperature and light) on the storage stability. Furthermore, centrifugation is used as the accelerated measurement of phase separation (Cortesi and Esposito, 2010).

\section{APPLICATION OF DOUBLE EMULSIONS LOADED WITH PLANT BIOACTIVES}

In this section, results of the recent studies considering the encapsulation of plant bioactives in double emulsions are presented.

Formation of double emulsions has enabled the co-delivery of hydrophobic curcumin and hydrophilic catechin, as synergistic bioactive compounds. Curcumin was dispersed in mixture of olive oil (oil phase) and PGPR (lipophilic emulsifier). Catechin was dissolved in inner water phase, including gelatin $(3 \%), \mathrm{NaCl}(2 \%)$, and ascorbic acid (0.2\%). Two-step emulsification method was used in order to prepare a $\mathrm{W} / \mathrm{O} / \mathrm{W}$ double emulsion and Tween 80 was used as a hydrophilic emulsifier. Encapsulation efficiency for curcumin and catechin was more than $88 \%$. Moreover, the encapsulation of catechin and curcumin within $\mathrm{W} / \mathrm{O} / \mathrm{W}$ emulsion has increased their stability and bioaccessibility significantly in simulated gastrointestinal fluid in the comparison to that of suspended curcumin and catechin solutions (Aditya et al., 2015).

Anthocyanins are a water-soluble phenolic compound with numerous health benefits. Furthermore, anthocyanin can be used as a renewable and sustainable source of plant-derived colorant. However, their use in commercial products is usually restricted due to poor chemical stability. In the study of Liu et al. (2019) the possibility of encapsulating anthocyanin in double emulsions is examined in order to improve anthocyanin stability. Primary (W/O) emulsion consisting $80 \%$ oil phase (corn oil and PGPR 5\%) and 20\% aqueous phase (anthocyanin in phosphate buffer) was prepared using a microfluidizer. Double (W/O/W) emulsion was formed by reemulsifieng of primary emulsion (20\%) aqueous phase consisting of quillaja saponin as hydrophilic emulsifier in phosphate buffer. It was concluded that the encapsulation of the anthocyanin in the inner aqueous phase of double emulsion appeared to reduce $\mathrm{pH}$-induced instability. The presence of anthocyanin has influenced emulsion formation, shape, size and structure of the $\mathrm{W} / \mathrm{O}$ droplets.

In order to prepare stable $\mathrm{W} / \mathrm{O} / \mathrm{W}$ emulsion, it is necessary to examine the influence of numerous process and formulation parameters. The formation and stabilization of $\mathrm{W} / \mathrm{O} / \mathrm{W}$ emulsion as a carrier for chlorophyllin and lemongrass essential oil was investigated (Artiga-Artigas et al., 2019). Firstly, it was concluded that the primary emulsion with better stability was obtained by using PGPR (4\%) as a lipophilic emulsifier than in the case of Span 80. Furthermore, optimal conditions for obtaining the primary emulsion with monomodal distribution by the high-share homogenization were $11000 \mathrm{rpm}$ for 5 minutes. The addition of sodium alginate $(2 \%)$ and sodium chloride $(0.05 \mathrm{M})$ in the aqueous phase of the primary emulsion was significant for the stabilization of emulsion. Secondly, it was concluded that lecithin was superior in comparison to the Tween 20, as a hydrophilic emulsifier of $\mathrm{W} / \mathrm{O} / \mathrm{W}$ emulsion since the polydispersity index was lower and stability during storage was better in case of double emulsions with lecithin. It was reported that after dispersing primary emulsion in outer water phase, high-share homogenization (5600 rpm, $2 \mathrm{~min}$ ) and magnetic stirring of obtained mixture (750 rpm during $24 \mathrm{~h}$ ) are considered as optimal procedure for obtaining stable double emulsion. In order to prevent migration of ions between the inner and outer water phase, the same concentration of sodium alginate and sodium chloride was added in the outer aqueous phase as in the inner aqueous phase. The results of this study have shown that higher encapsulation efficiency of chlorophyllin was achieved in emulsions with lemongrass essential oil than in those without. Consequently, it was concluded that a small quantity of lemongrass essential oil was able to slow down the inner water droplet diffusion.

Double $(\mathrm{W} / \mathrm{O} / \mathrm{W})$ emulsions were used as carriers for resveratrol in order to improve its bioavailability (Wang et al., 2017). Emulsions were prepared by using the high-pressure homogenization. Influence of various lipophilic (Span 80, PGPR, glyceryl monostearate, lecithin) and hydrophilic (peanut protein isolate, modified starch, Tween 80 , chitosan, protein-mannose Maillard reaction products, pectin, protein-mannose mixture, whey-protein isolate) emulsifiers was investigated. Moreover, the effects of the emulsifier concentration, oil phase-internal water phase ratio and homogenization pressure on the microstructure, droplet size, distribution, zeta potential, viscosity and encapsulation efficiency of $\mathrm{W} / \mathrm{O} / \mathrm{W}$ emulsions were considered. It was shown that with PGPR $(10 \%)$ as a lipophilic emulsifier and Tween $80(5 \%)$ as a hydrophilic emulsifier stable double emulsion can be formulated. The optimal oil phaseinternal water phase ratio was 80:20 and homogenization pressures in the first and second step were $30 \mathrm{MPa}$ and $10 \mathrm{MPa}$ respectively. Resveratrol $(0.04 \%)$ was encapsulated in the inner water phase of $\mathrm{W} / \mathrm{O} / \mathrm{W}$ emulsion with an encapsulation efficiency of $99.97 \%$.

There are many studies which are considering the replacement of synthetic emulsifiers with natural biomolecules such as hydrocolloids (gum arabic, pectin, sodium alginate, etc.) and proteins. Recently, it was reported that pectin- whey protein concentrate (WPC) complex can be the appropriate stabilizer for $\mathrm{W} / \mathrm{O} / \mathrm{W}$ emulsion loaded with bioactive compounds such as gallic acid (Gharehbeglou et al., 2019a). Furthermore, it was shown that the effect of pectin-WPC complex and Tween 80 against coalescence and creaming are the same. The use of 
Tween 80 is related to the potential health risks and because of this, it is reasonable to use natural biomolecules such as pectin-WPC complex. The formulation of WPC-pectin double $\mathrm{W} / \mathrm{O} / \mathrm{W}$ nano-emulsions with oleuropein was also optimized by considering Span 80 (lipophilic emulsifier) content, WPC and pectin content, the ratio of inner to outer phase and $\mathrm{pH}$ (Gharehbeglou et al., 2019b). Optimal conditions were found to be $8.74 \%$ Span, $8 \%$ WPC, $1.97 \%$ pectin, $1: 4$ ratio of inner to outer phase and $\mathrm{pH}$ 6.1. Samples were prepared by sonication and droplets size was $191 \mathrm{~nm}$, zeta potential was $-26.8 \mathrm{mV}$, and also encapsulation efficiency was high (91\%).

Mehrnia et al. (2017) have investigated the influence of novel biopolymer angum gum (exudates of almond tree) on the stability of crocin-loaded double emulsions. Crocin is a highly water-soluble carotenoid, sensitive to environmental conditions such as light, oxygen and $\mathrm{pH}$. In that regard, double emulsions are used in order to protect crocin during processing and storage. It is reported that angum gum had lower emulsifying capacity than gum arabic and whey protein isolate and it is related to the bigger droplet size and broader spans. On the other hand, emulsions with angum gum are characterized by better stability due to the higher viscosity and gel-like structure of emulsions with angum gum.

In the few studies in vitro digestion tests were used in order to understand physicochemical changes of $\mathrm{W} / \mathrm{O} / \mathrm{W}$ emulsions and release of bioactive compounds during digestion. Double emulsion with water extract of red beet was prepared with rapeseed oil as the oil phase, PGPR and polar lipid fraction from oat were used as lipophilic and hydrophilic emulsifiers, respectively. Pancreatic lipase was used for simulation of intestinal phase of digestion and prepared emulsion was evaluated in order to examine release of betalain and emulsion structure during digestion. Betalain release during the first 120 minutes was fast, but after that, upon 180 minutes, there was no further release. The final amount released was approximately $35 \%$. It was demonstrated that release of betalain was influenced by the structure and size of droplets. The release of betalain was induced by coalescence of inner droplets. On the other hand, release was reduced as a consequence of outer droplets aggregation. It was concluded that the release from double $(\mathrm{W} / \mathrm{O} / \mathrm{W})$ emulsion was controlled by destabilization mechanisms (Kaimainen et al., 2015).

The bioactivity of anthocyanins can be reduced due to digestion. Therefore, the in vitro digestion model was used as a tool for understanding the physicochemical changes and controlled release of encapsulated grape skin extract from double emulsions stabilized by PGPR and soy protein isolates. It was demonstrated that the microstructure of droplets was not disturbed by mouth digestion ( $\alpha$-amylase). Upon gastric phase approximately $6 \%$ of anthocyanins ware released, and this result could be related to the pepsin activity and collapse of droplet structure. Anthocyanins were released predominantly during the intestinal phase as result of pancreatin and lipase digestion of the lipid layer (Xu et al., 2018).

Double emulsions with apigenin as a common bioactive flavonoid with potential health benefits were formulated in order to overcome problem with delivery of this bioactive molecule due to its low water solubility. Properties (droplet size, morphology, zeta potential) of apigenin-loaded emulsions were investigated by considering simulated mouth, gastric and intestinal phase of digestion. It is reported that apigenin-loaded soybean oil-Tween 80 emulsions have kept stability during simulated digestion. Furthermore, the in vivo pharmacokinetic study has shown that oral bioavailability of apigenin encapsulated in double emulsion has increased approximately nine-fold in the comparison to the apigenin suspension (Kim et al., 2016).

In the study presented by Toledo-Madrid et al. (2018) microen- capsulation of purple cactus pear fruit extract by spray drying of $\mathrm{W} / \mathrm{O} / \mathrm{W}$ emulsions was compared with conventional spray drying of the extract with maltodextrin as a carrier. Double emulsion was formed by re-emulsifying the primary $(\mathrm{W} / \mathrm{O})$ emulsion in a solution of whey protein isolates $(10 \%)$ by using Ultra Turrax homogenizer. Primary emulsion (W/O) was obtained through high-share homogenization of inner water phase (85\% concentrated purple cactus pear fruit and 15\% glycerol) and oil phase (87\% canola oil and 13\% PGPR). It was concluded that lower encapsulation and retention efficiency was achieved by spray drying of $\mathrm{W} / \mathrm{O} / \mathrm{W}$ emulsions, however particles obtained by spray drying of $\mathrm{W} / \mathrm{O} / \mathrm{W}$ emulsions are a promising controlled-delivery vehicle for antioxidant compounds.

In order to achieve sustained release of Cecropia glaziovii extract with vasorelaxant effect, $\mathrm{W} / \mathrm{O} / \mathrm{W}$ emulsions were formulated, followed by solvent evaporation/extraction. Firstly, the influence of four formulation parameters was analyzed, including the amount and type of poly lactic-co-glycolic acid-PLGA, amount of $C$. glaziovii extract and extraction phase volume. After optimization of those factors, considering particle size, size distribution and encapsulation efficiency (EE), microparticles with low encapsulation efficiency were obtained. In order to improve $\mathrm{EE}$, in the second experimental design osmotic pressure of the external phase was optimized by adding $\mathrm{NaCl}$. It was concluded that the addition of $\mathrm{NaCl}$ has influenced particle surface structure, leading to the formation of denser and less porous particles. Furthermore, the sustained release of C. glaziovii extract from microparticles was achieved, which can result in a long-lasting relaxation effect (dos Santos et al., 2018).

Double W/O/W emulsions have also been used for the production of lipid nanoparticles with hydrophilic bioactive compounds. In the article presented by Pimentel-Moral et al. (2019), nanostructured lipid carriers (NLC) loaded with extracts of Hibiscus sabdariffa were produced by combining double emulsion and ultrasonication techniques. The optimal NLC formulation composed of 2.21\% lipid phase (mixture of Soybean oil and Biograpress ${ }^{\mathrm{TM}}$ Vegetal BM 297 ATO) and $1.93 \%$ surfactants (Tween 80 and Span 80 ) was characterized by mean particle size $(107 \pm 0.4 \mathrm{~nm})$ polydispersity index $(0.163 \pm 0.010)$, zeta potential $(-25.8 \pm 0.9 \mathrm{mV})$.

\section{CONCLUSION}

Double emulsions are advanced delivery systems with numerous applications. In that regard, the double emulsions are used for encapsulation, target and controlled delivery of plant bioactive compounds. Instability has been the main issue, but the stability of the multiple emulsions has been improved by steric stabilization, mechanical stabilization, depletion stabilization. In the last decade, biopolymer complexes and conjugates are usually used as natural emulsifiers and stabilizers for double emulsions with plant bioactive compounds. Furthermore, strategies for improvement of encapsulation efficiency and release modification are extensively examined.

\section{ACKNOWLEDGMENTS}

This research was supported by the Ministry of Education, Science and Technological Development of Republic of Serbia grant numbers TR 34007 and 46013.

\section{REFERENCES}

Aditya, N., Aditya, S., Yang, H., Kim, H. W., Park, S. O. and Ko, S. (2015). Co-delivery of hydrophobic curcumin and hydrophilic catechin by a water-in-oil-in-water double emulsion, Food Chemistry 173: 7-13. 
Akhtar, M., Murray, B. S., Afeisume, E. I. and Khew, S. H. (2014). Encapsulation of flavonoid in multiple emulsion using spinning disc reactor technology, Food Hydrocolloids 34: 62-67.

Artiga-Artigas, M., Molet-Rodríguez, A., Salvia-Trujillo, L. and Martín-Belloso, O. (2019). Formation of double (W1/O/W2) emulsions as carriers of hydrophilic and lipophilic active compounds, Food and Bioprocess Technology 12(3): 422-435.

Aserin, A. (2008). Multiple Emulsion: Technology and Applications, John Wiley \& Sons.

Ashjari, M., Khoee, S. and Mahdavian, A. R. (2012). A multiple emulsion method for loading 5-fluorouracil into a magnetite-loaded nanocapsule: a physicochemical investigation, Polymer International 61(5): 850-859.

Benichou, A., Aserin, A. and Garti, N. (2004). Double emulsions stabilized with hybrids of natural polymers for entrapment and slow release of active matters, Advances in Colloid and Interface Science 108-109: 29-41.

Berendsen, R. (2014). Encapsulation of procyanidins in double emulsions stabilized by protein-polysaccharide complexes, Ph.D. Thesis, Universitat Rovira i Virgili.

Bernewitz, R., Dalitz, F., Köhler, K., Schuchmann, H. and Guthausen, G. (2013). Characterisation of multiple emulsions by NMR spectroscopy and diffusometry, Microporous and Mesoporous Materials 178: 69-73.

Bhatia, N., Pandit, S., Agrawal, S. and Gupta, D. (2013). A Review On Multiple Emulsions, International Journal of Pharmaceutical Erudition p. 9.

Carrillo-Navas, H., Cruz-Olivares, J., Varela-Guerrero, V., Alamilla-Beltrán, L., Vernon-Carter, E. J. and Pérez-Alonso, C. (2012). Rheological properties of a double emulsion nutraceutical system incorporating chia essential oil and ascorbic acid stabilized by carbohydrate polymer-protein blends, Carbohydrate Polymers 87(2): 1231-1235.

Champagne, C. P. and Fustier, P. (2007). Microencapsulation for the improved delivery of bioactive compounds into foods, Current Opinion in Biotechnology 18(2): 184-190.

Chung, C., Smith, G., Degner, B. and McClements, D. J. (2016). Reduced fat food emulsions: physicochemical, sensory, and biological aspects, Critical Reviews in Food Science and Nutrition 56(4): 650-685.

Cortesi, R. and Esposito, E. (2010). Pharmaceutical and biotechnological applications of multiple emulsions, in M. Fanun (ed.), Colloids in Drug Delivery, Vol. 20100581, CRC Press, pp. 203-220.

Dluska, E., Markowska-Radomska, A., Metera, A., Tudek, B. and Kosicki, K. (2017). Multiple emulsions as effective platforms for controlled anti-cancer drug delivery, Nanomedicine 12(18): 2183-2197.

dos Santos, T. C., Battisti, M. A., Lobo, K. L., Caon, T., Linder, A. E., Sonaglio, D. and de Campos, A. M. (2018). Vasorelaxant effect of standardized extract of Cecropia glaziovii Snethl encapsulated in PLGA microparticles: In vitro activity, formulation development and release studies, Materials Science and Engineering: C 92: 228-235.

Garti, N., Frenkel, M. and Shwartz, R. (1983). Multiple emulsions. part II: Proposed technique to overcome unpleasant taste of drugs, Journal of Dispersion Science and Technology 4(3): 237-252.
Gharehbeglou, P., Jafari, S. M., Hamishekar, H., Homayouni, A. and Mirzaei, H. (2019a). Pectin-whey protein complexes vs. small molecule surfactants for stabilization of double nano-emulsions as novel bioactive delivery systems, Journal of Food Engineering 245: 139-148.

Gharehbeglou, P., Jafari, S. M., Homayouni, A., Hamishekar, H. and Mirzaei, H. (2019b). Fabrication of double W1/O/W2 nano-emulsions loaded with oleuropein in the internal phase (W1) and evaluation of their release rate, Food Hydrocolloids 89: 44-55.

Giri, T. K., Choudhary, C., Ajazuddin, Alexander, A., Badwaik, H. and Tripathi, D. K. (2013). Prospects of pharmaceuticals and biopharmaceuticals loaded microparticles prepared by double emulsion technique for controlled delivery, Saudi Pharmaceutical Journal 21(2): 125-141.

Iqbal, M., Zafar, N., Fessi, H. and Elaissari, A. (2015). Double emulsion solvent evaporation techniques used for drug encapsulation, International Journal of Pharmaceutics 496(2): 173190.

Jiménez-Colmenero, F. (2013). Potential applications of multiple emulsions in the development of healthy and functional foods, Food Research International 52(1): 64-74.

Kaimainen, M., Marze, S., Järvenpää, E., Anton, M. and Huopalahti, R. (2015). Encapsulation of betalain into $\mathrm{w} / \mathrm{o} / \mathrm{w}$ double emulsion and release during in vitro intestinal lipid digestion, LWT - Food Science and Technology 60(2): 899-904.

Kim, B.-K., Cho, A.-R. and Park, D.-J. (2016). Enhancing oral bioavailability using preparations of apigenin-loaded $\mathrm{W} / \mathrm{O} / \mathrm{W}$ emulsions: In vitro and in vivo evaluations, Food Chemistry 206: 85-91.

Lamba, H., Sathish, K. and Sabikhi, L. (2015). Double emulsions: emerging delivery system for plant bioactives, Food and Bioprocess Technology 8(4): 709-728.

Liu, J., Tan, Y., Zhou, H., Muriel Mundo, J. L. and McClements, D. J. (2019). Protection of anthocyanin-rich extract from $\mathrm{pH}$-induced color changes using water-in-oil-in-water emulsions, Journal of Food Engineering 254: 1-9.

McClements, D. J., Decker, E. A., Park, Y. and Weiss, J. (2009). Structural design principles for delivery of bioactive components in nutraceuticals and functional foods, Critical Reviews in Food Science and Nutrition 49(6): 577-606.

Mehrnia, M.-A., Jafari, S.-M., Makhmal-Zadeh, B. S. and Maghsoudlou, Y. (2017). Rheological and release properties of double nano-emulsions containing crocin prepared with Angum gum, Arabic gum and whey protein, Food Hydrocolloids 66: 259-267.

Mezzenga, R., Folmer, B. M. and Hughes, E. (2004). Design of double emulsions by osmotic pressure tailoring, Langmuir 20(9): 3574-3582.

Morais, A. R. d. V., Alencar, v. d. N., Xavier Júnior, F. H., Oliveira, C. M. d., Marcelino, H. R., Barratt, G., Fessi, H., Egito, E. S. T. d. and Elaissari, A. (2016). Freeze-drying of emulsified systems: A review, International Journal of Pharmaceutics 503(1-2): 102-114.

Muschiolik, G. and Dickinson, E. (2017). Double emulsions relevant to food systems: preparation, stability, and applications, Comprehensive Reviews in Food Science and Food Safety 16(3): 532-555. 
Pal, R. (2011). Rheology of simple and multiple emulsions, Current Opinion in Colloid E Interface Science 16(1): 41-60.

Patravale, V. B. and Mandawgade, S. D. (2008). Novel cosmetic delivery systems: an application update, International Journal of Cosmetic Science 30(1): 19-33.

Paul, S., Kumar, A., Yedurkar, P. and Sawant, K. (2013). Design and development of multiple emulsion for enhancement of oral bioavailability of acyclovir, Drug Development and Industrial Pharmacy 39(11): 1809-1817.

Pimentel-Moral, S., Teixeira, M., Fernandes, A., Borrás-Linares, I., Arráez-Román, D., Martínez-Férez, A., Segura-Carretero, A. and Souto, E. (2019). Polyphenols-enriched Hibiscus sabdariffa extract-loaded nanostructured lipid carriers (NLC): Optimization by multi-response surface methodology, Journal of Drug Delivery Science and Technology 49: 660-667.

Raviadaran, R., Muthoosamy, K. and \& Manickam, S. (2018). Simple and multiple emulsions emphasizing on industrial applications and stability assessment, Process Engineering and Quality Assurance, Apple Academic Press, pp. 179-230.

Salazar-Miranda, M. A., Cruz-Sosa, F., Rodríguez-Huezo, M. E., Jiménez-Alvarado, R. and Pérez-Alonso, C. (2016). Microencapsulation of chlorthalidone by spray-drying of double emulsion and melt granulation coating, Drying Technology 34(9): 1118-1128.

Schmidts, T., Dobler, D., Nissing, C. and Runkel, F. (2009). Influence of hydrophilic surfactants on the properties of multiple $\mathrm{W} / \mathrm{O} / \mathrm{W}$ emulsions, Journal of Colloid and Interface Science 338(1): 184-192.

Schuch, A., Deiters, P., Henne, J., Köhler, K. and Schuchmann, H. P. (2013). Production of W/O/W (water-in-oil-in-water) multiple emulsions: droplet breakup and release of water, Journal of Colloid and Interface Science 402: 157-164.

Surh, J., Vladisavljević, G. T., Mun, S. and McClements, D. J. (2007). Preparation and characterization of water/oil and water/oil/water emulsions containing biopolymer-gelled water droplets, Journal of Agricultural and Food Chemistry 55(1): 175-184.

Talegaonkar, S. and Vyas, S. (2005). Inverse targeting of diclofenac sodium to reticuloendothelial system-rich organs by sphere-in-oil-in-water (s/o/w) multiple emulsion containing poloxamer 403, Journal of Drug Targeting 13(3): 173178.

Toledo-Madrid, K., Gallardo-Velázquez, T. and Osorio-Revilla, G. (2018). Microencapsulation of purple cactus pear fruit (Opuntia ficus indica) Eextract by the combined method $\mathrm{W} / \mathrm{O} / \mathrm{W}$ double emulsion-spray drying and conventional spray drying: A comparative study, Processes 6(10): 189.

van der Graaf, S., Schroen, C. and Boom, R. (2005). Preparation of double emulsions by membrane emulsification - a review, Journal of Membrane Science 251(1-2): 7-15.

Vasiljevic, D., Parojcic, J., Primorac, M. and Vuleta, G. (2006). An investigation into the characteristics and drug release properties of multiple $\mathrm{W} / \mathrm{O} / \mathrm{W}$ emulsion systems containing low concentration of lipophilic polymeric emulsifier, International Journal of Pharmaceutics 309(1-2): 171-177.

Vladisavljević, G., Al Nuumani, R. and Nabavi, S. (2017). Microfluidic production of multiple emulsions, Micromachines 8(3): 75 .
Vladisavljević, G. T., Kobayashi, I. and Nakajima, M. (2012). Production of uniform droplets using membrane, microchannel and microfluidic emulsification devices, Microfluidics and Nanofluidics 13(1): 151-178.

Wang, J., Shi, A., Agyei, D. and Wang, Q. (2017). Formulation of water-in-oil-in-water $(\mathrm{W} / \mathrm{O} / \mathrm{W})$ emulsions containing trans-resveratrol, RSC Advances 7(57): 35917-35927.

Xu, W., Yang, Y., Xue, S. J., Shi, J., Lim, L.-T., Forney, C., Xu, G. and Bamba, B. S. B. (2018). Effect of in vitro digestion on water-in-iil-in-water emulsions containing anthocyanins from grape skin powder, Molecules 23(11). 